\title{
CORRECTION TO: MOTIVIC DECOMPOSITION OF PROJECTIVE PSEUDO-HOMOGENEOUS VARIETIES
}

\author{
SRIMATHY SRINIVASAN* \\ School of Mathematics \\ Institute for Advanced Study \\ Princeton, NJ 08540, USA \\ srimathy@ias.edu
}

Correction to: Transformation Groups (2017) 22(4): 1125-1142

Abstract. We point out an error in the proof of a lemma in [Sri17] and correct it by proving a stronger version of the lemma using a theorem of Pierre Deligne.

It was pointed out by Pierre Deligne that the proof of Lemma 6.2 in [Sri17] is incorrect. Although the statement of the lemma is correct, here we state a stronger version of the lemma and prove it using a result of P. Deligne [Del18]. This gives an easy proof of Corollary 6.3 in [Sri17] which we also comment on. The rest of the paper is unaffected.

First we will describe the error in the proof of Lemma 6.2 . Recall that $\tilde{X}$ is a projective pseudo-homogeneous variety for $G$ and $X$ is the corresponding projective homogeneous variety. The base field of these varieties, denoted by $k$, is assumed to be perfect. The lemma claims that for any field extension $F$ of $k, X$ has an $F$-point if and only if $\widetilde{X}$ has an $F$-point. The "only if" direction is clear because of the existence of canonical $G$-equivariant $k$-morphism $X \rightarrow \widetilde{X}$ obtained via the universal property of quotients(see below). The proof of the "if " direction cites Exercise 13.2.5(4) in [Spr09] and wrongly concludes that the Tits index of $G$ over $F$ and $F^{\prime}$ are the same where $F^{\prime}$ is the perfect closure of $F$. The conclusion is wrong because the exercise implies that $T_{s, F^{\prime}}=T_{s, F}$ where for an inseparable extension $E$ of $F$ and for an $F$-torus $T, T_{s, E}$ denotes the unique maximal $E$-split subtorus of $T$. This does not necessarily mean that the Tits index of $G$ over $F$ and $F^{\prime}$ are the same, as the field extension $F^{\prime} / F$ could possibly give rise to a different $F^{\prime}$-split torus of larger rank in $G$ that is not necessarily contained in the torus $T$ that we started with.

We now fix this error by replacing the lemma with a stronger version and give a proof.

DOI: $10.1007 / \mathrm{s} 00031-019-09539-2$

The online version of the original article can be found at https://doi.org/10.1007/ s00031-016-9407-4.

*This material is based upon work supported by the National Science Foundation under Grant No. DMS-1638352.

Received June 4, 2019. Accepted June 14, 2019.

Published online July 30, 2019.

Corresponding Author: Srimathy Srinivasan, e-mail: srimathy@ias.edu 
Lemma 1 (Stronger version of Lemma 6.2 in [Sri17]). There exist $k$-morphisms $f: X \rightarrow \widetilde{X}$ and $g: \widetilde{X} \rightarrow X$.

Proof. With notations as in the paper, recall that $X_{K} \simeq G / P$ and $\widetilde{X}_{K} \simeq G / \widetilde{P}$ where $K$ is the algebraic closure of $k$ (which is assumed to be perfect). Since $P \hookrightarrow \widetilde{P}$, by the universal property of quotients, there exists a unique $G$-equivariant morphism $\mathcal{F}: G / P \rightarrow G / \widetilde{P}$ over $K$. We now use the following descending argument to get a $k$-morphism $f$. The uniqueness of $\mathcal{F}$ together with the fact that the $G$-action on $X$ and $\widetilde{X}$ is defined over $k$, implies that $\mathcal{F}$ is $\mathcal{G}_{k}$ invariant where $\mathcal{G}_{k}$ is the absolute Galois group of $k$. Hence the $K$-morphism $\mathcal{F}$ descends to a $k$-morphism $f: X \rightarrow \widetilde{X}$. To get a $k$-morphism from $\widetilde{X}$ to $X$ we proceed as follows. Let $G^{(n)}: G \times$ Frob $^{n} k$ denote the $n$-th order Frobenius twist of $G$, i.e., $G^{(n)}$ as a $k$-scheme has the same underlying topological space as $G$ but has a $k$-structure twisted by the ring homomorphism $a \mapsto \sqrt[p]{a}$. Then $G^{(n)}$ is an algebraic group of the same type as $G$ and the $n$-th order Frobenius induces a surjective $k$-morphism of algebraic groups

$$
\operatorname{Frob}^{n}: G \rightarrow G^{(n)}
$$

with kernel $G_{n}$ yielding a $k$-isomorphism of algebraic groups

$$
G / G_{n} \simeq G^{(n)} .
$$

Now by [Lau93, §2], there is an embedding

$$
\widetilde{P} \hookrightarrow G_{m} P
$$

for some large enough $m$. Recall that by Deligne ([Del18]), there exists $n \geq m$ such that $G^{(n)}$ is $k$-isomorphic to $G$. Call this isomorphism $\phi$. Then $X$ is projective homogeneous for $G$ where $G$ acts via the $k$-morphism

$$
G \rightarrow G / G_{n} \simeq G^{(n)} \stackrel{\phi}{\rightarrow} G
$$

Over $K$ this implies that $X_{K} \simeq G / G_{n} P$. Since $n \geq m$, by the universal property of quotients we get a unique $G$-equivariant map

$$
G / \widetilde{P} \rightarrow G / G_{n} P
$$

Using the descending argument as above we get $k$-morphism

$$
g: \tilde{X} \rightarrow X
$$

The proof of [Sri17, Cor. 6.3] can now be easily derived as follows.

Corollary 2 ([Sri17, Cor. 6.3]). Let $X$ and $\widetilde{X}$ be as above. Then in Chow $(k, \Lambda)$, $U_{X} \simeq U_{\widetilde{X}}$.

Proof. By [Kar13, Cor. 2.15], it suffices to show multiplicity one correspondences $\alpha: \mathcal{M}(X) \rightarrow \mathcal{M}(\tilde{X})$ and $\beta: \mathcal{M}(\tilde{X}) \rightarrow \mathcal{M}(X)$. Take $\alpha$ and $\beta$ respectively to be the correspondence induced from $k$-morphisms $f$ and $g$ constructed in Lemma 6.2 . 


\section{References}

[Del18] P. Deligne, Extension des scalaires par le morphisme de Frobenius, pour les groupes réductifs, C. R. Math. Acad. Sci. Paris 356 (2018), no. 7, 717-719.

[Kar13] N. A. Karpenko, Upper motives of algebraic groups and incompressibility of Severi-Brauer varieties, J. Reine Angew. Math. 677 (2013), 179-198.

[Lau93] N. Lauritzen, Splitting properties of complete homogeneous spaces, J. Algebra 162 (1993), no. 1, 178-193.

[Spr09] T. A. Springer, Linear Algebraic Groups, 2nd Ed., Modern Birkhäuser Classics, Birkhäuser Boston, Boston, MA, 2009.

[Sri17] S. Srinivasan, Motivic decomposition of projective pseudo-homogeneous varieties, Transform. Groups 22 (2017), no. 4, 1125-1142. 